Göteborg ITP 98-08

\title{
Dualities between Poisson brackets and antibrackets
}

\author{
Igor Batalin円 and Robert Marnelius \\ Institute of Theoretical Physics \\ Chalmers University of Technology \\ Göteborg University \\ S-412 96 Göteborg, Sweden
}

\begin{abstract}
Recently it has been shown that antibrackets may be expressed in terms of Poisson brackets and vice versa for commuting functions in the original bracket. Here we also introduce generalized brackets involving higher antibrackets or higher Poisson brackets where the latter are of a new type. We give generating functions for these brackets for functions in arbitrary involutions in the original bracket. We also give master equations for generalized Maurer-Cartan equations. The presentation is completely symmetric with respect to Poisson brackets and antibrackets.
\end{abstract}

\footnotetext{
${ }^{1}$ On leave of absence from P.N.Lebedev Physical Institute, 117924 Moscow, Russia E-mail: batalin@td.lpi.ac.ru.

${ }^{2}$ E-mail: tferm@fy.chalmers.se
} 


\section{Introduction.}

Poisson brackets and antibrackets are two basic binary operations in classical dynamics. Poisson brackets have an old history and are crucial for the transition to the quantum theory. Antibrackets [1, 2] on the other hand play a crucial role in the BV quantization of general gauge theories [2]. Recently we have constructed a consistent quantum antibracket corresponding to the commutator for Poisson brackets [3]. This construction did not only cast new light on the BV quantization but also indirectly on the relations between antibrackets and Poisson brackets. The purpose of the present paper is to communicate these relations. The presentation will be completely symmetric with respect to the two brackets and will therefore in a natural way also include the results in [4, 5, 6]. In this way we demonstrate the duality between the two brackets in the following sense: To any relation involving both brackets there is a dual relation in which the brackets are interchanged. As a result we present new higher Poisson brackets constructed in complete analogy with higher antibrackets. Also the recently given quantum master equation for generalized Maurer-Cartan equations given in [7] is shown to have a dual master equation at the classical level.

On a manifold, $\mathcal{E}$, with dimension $(2 n, 2 n)$ we may have either a nondegenerate Poisson bracket or a nondegenerate antibracket. (The defining properties of these brackets are given in appendices $\mathrm{A}$ and $\mathrm{B}$.) If $\mathcal{E}$ is a symplectic manifold we have a Poisson bracket and the (local) Darboux coordinates with their Grassmann parities $(\varepsilon=0,1 \bmod 2)$ may be defined to be

$$
\begin{aligned}
& z^{A} \equiv\left\{x^{a}, x_{a}^{*}, p_{a}, p_{*}^{a}\right\}, \quad a=1, \ldots, n, \\
& \varepsilon_{a} \equiv \varepsilon\left(x^{a}\right)=\varepsilon\left(p_{a}\right), \quad \varepsilon\left(x_{a}^{*}\right)=\varepsilon\left(p_{*}^{a}\right)=\varepsilon_{a}+1 .
\end{aligned}
$$

In terms of these Darboux coordinates the Poisson bracket has the nonzero elements

$$
\left\{x^{a}, p_{b}\right\}=\delta_{b}^{a}=-(-1)^{\varepsilon_{a}}\left\{p_{b}, x^{a}\right\}, \quad\left\{x_{a}^{*}, p_{*}^{b}\right\}=\delta_{a}^{b}=(-1)^{\varepsilon_{a}}\left\{p_{*}^{b}, x_{a}^{*}\right\} .
$$

Similarly, if $\mathcal{E}$ is an antisymplectic manifold we have an antibracket and the (local) Darboux coordinates (1.1) in terms of which the antibracket has the nonzero elements

$$
\left(x^{a}, x_{b}^{*}\right)=\delta_{b}^{a}=-\left(x_{b}^{*}, x^{a}\right), \quad\left(p_{a}, p_{*}^{b}\right)=\delta_{a}^{b}=-\left(p_{*}^{b}, p_{a}\right) .
$$

One basic question is whether or not one may have both a Poisson bracket and an antibracket on $\mathcal{E}$. Our treatment here will lead to a negative answer to this question if the brackets are the conventional ones. However, we will display some generalized possibilities of coexistence.

As an introductory remark let us for a moment assume that it is possible to have both brackets on $\mathcal{E}$. The question is then how they are supposed to be related. In [8, 9] it was proposed that the equations of motions should be possible to write down both in terms of Poisson brackets and in terms of antibrackets. There should then exist two Hamiltonians, one even $(H)$ and one odd $(Q)$ related by

$$
\{f(z), H\}=(f(z), Q) .
$$

However, this condition turns out to be very restrictive and allows only for very special forms of $H$ and $Q$ [8, 9]. Such relations are therefore not valid in general. On the other 
hand, it is amusing to note that if the two brackets for some reasons allow for the common Darboux coordinates (1.1) then there exists a very natural solution of (1.4), namely

$$
Q=p_{a} p_{*}^{a}(-1)^{\varepsilon_{a}}-x^{a} x_{a}^{*}(-1)^{\varepsilon_{a}}, \quad H=-x^{a} p_{a}-x_{a}^{*} p_{*}^{a} .
$$

This solution satisfies the following supersymmetric Poisson bracket algebra and antibracket algebra which seems to be a natural consequence of (1.4) 8, 9.

$$
\begin{aligned}
& \{H, H\}=\{H, Q\}=0, \quad\{Q, Q\}=2 H \\
& (Q, Q)=(H, Q)=0, \quad(H, H)=2 Q .
\end{aligned}
$$

In addition we have the relations

$$
\begin{aligned}
& \left(z^{A}, z^{B}\right)=\left\{z^{A},\left\{Q, z^{B}\right\}\right\}=\frac{1}{2}\left(\left\{z^{A},\left\{Q, z^{B}\right\}\right\}-\left\{z^{B},\left\{Q, z^{A}\right\}\right\}(-1)^{\left(\varepsilon_{A}+1\right)\left(\varepsilon_{B}+1\right)}\right), \\
& \left\{z^{A}, z^{B}\right\}=\left(z^{A},\left(H, z^{B}\right)\right)=\frac{1}{2}\left(\left(z^{A},\left(H, z^{B}\right)\right)-\left(z^{B},\left(H, z^{A}\right)\right)(-1)^{\varepsilon_{A} \varepsilon_{B}}\right)
\end{aligned}
$$

where $z^{A}$ are the Darboux coordinates (1.1). However, the solution of the coexistence problem is not that simple. In fact, there exists no relation between Poisson brackets and antibrackets for arbitrary functions of $z^{A}$ of the form (1.7) with $Q$ and $H$ given by (1.5). On the other hand in [3]-[6] it has been demonstrated that there exist possible ways to express antibrackets in terms of Poisson brackets and vice versa but then for subclasses of functions. Although this construction is similar to (1.7) the basic functions $Q$ and $H$ do not satisfy (1.6). In this paper we present these possible ways to express antibrackets in terms of Poisson brackets and vice versa. In order to do this on the whole manifolds $\mathcal{E}$ we are then naturally led to consider generalized brackets defined in a definite way.

In section 2 we present the results in [3]-[6] on how ordinary antibrackets may be expressed in terms of ordinary Poisson brackets and vice versa. This can only be done for the subclass of functions satisfying an abelian algebra in the original bracket. We also give the precise circumstances under which (1.4) is valid for arbitrary $H$ or $Q$. In section 3 we present generating functions for Poisson brackets and antibrackets for functions satisfying a Lie algebra in the original brackets which naturally lead us to generalized brackets involving higher antibrackets and higher Poisson brackets which are valid on the whole manifold $\mathcal{E}$. In section 4 generating functions of brackets of functions in arbitrary involutions in the original bracket are presented. We demonstrate the existence of two dual master equations for generalized Maurer-Cartan equations which naturally involve the generalized brackets in section 3. In section 5 we then conclude the paper. In two appendices we give the defining properties of the conventional Poisson brackets and antibrackets.

\section{Antibrackets in terms of Poisson brackets and vice versa.}

Here we review the results in [3]- [6] in a unified manner which illuminates the possible ways to express antibrackets in terms of Poisson brackets and vice versa. The presentation is in the spirit of [3].

Assume the existence of a graded Poisson bracket or a graded antibracket on the $(2 n, 2 n)$-dimensional manifold $\mathcal{E}$. In terms of these nondegenerate brackets we define the following binary operations

$$
(f, g)_{Q} \equiv \frac{1}{2}\left(\{f,\{Q, g\}\}-\{g,\{Q, f\}\}(-1)^{\left(\varepsilon_{f}+1\right)\left(\varepsilon_{g}+1\right)}\right),
$$




$$
\{f, g\}_{R} \equiv \frac{1}{2}\left((f,(R, g))-(g,(R, f))(-1)^{\varepsilon_{f} \varepsilon_{g}}\right),
$$

where $Q$ is an odd function and $R$ an even function. These expressions satisfy all the properties of a conventional antibracket and a conventional Poisson bracket except for the Jacobi identies and Leibniz' rule (see appendices A and B). It is easily seen that e.g. Leibniz' rule require us to restrict the class of functions. Instead of Leibniz' rules (A.5) and $(\mathrm{B.5})$ we have

$$
\begin{aligned}
&(f g, h)_{Q}-f(g, h)_{Q}-(f, h)_{Q} g(-1)^{\varepsilon_{g}\left(\varepsilon_{h}+1\right)}= \\
&= \frac{1}{2}\{f, h\}\{g, Q\}(-1)^{\varepsilon_{h}\left(\varepsilon_{g}+1\right)}+\frac{1}{2}\{f, Q\}\{g, h\}(-1)^{\varepsilon_{g}}, \\
&\{f g, h\}_{R}-f\{g, h\}_{R}-\{f, h\}_{R} g(-1)^{\varepsilon_{g} \varepsilon_{h}}= \\
&=-\frac{1}{2}(f, h)(R, g)(-1)^{\varepsilon_{h}\left(\varepsilon_{g}+1\right)}-\frac{1}{2}(R, f)(g, h)(-1)^{\varepsilon_{g}+\varepsilon_{h}} .
\end{aligned}
$$

From these relations it follows that Leibniz' rule is valid for $(f, g)_{Q}$ if we restrict ourselves to the class of functions commuting in the original Poisson bracket on $\mathcal{E}$ (denoted $\mathcal{M} \subset \mathcal{E}$ in the following), and that it is valid for $\{f, g\}_{R}$ if we restrict ourselves to the class of functions commuting in the original antibracket on $\mathcal{E}$ (denoted $\mathcal{M}^{*} \subset \mathcal{E}$ in the following). On these submanifolds (2.1) and (2.2) reduce to (cf.[3]-[6] and (1.7))

$$
\begin{gathered}
(f, g)_{Q}=\{f,\{Q, g\}\} \quad \forall f, g \in \mathcal{M} \quad(\Leftrightarrow\{f, g\}=0), \\
\{f, g\}_{R}=(f,(R, g)) \quad \forall f, g \in \mathcal{M}^{*} \quad(\Leftrightarrow(f, g)=0) .
\end{gathered}
$$

In order for the expressions (2.1) and (2.2) to also satisfy the Jacobi identities we have to impose the additional conditions [3, 6]

$$
\begin{gathered}
\{\{f,\{g,\{h, Q\}\}\}, Q\}-\frac{1}{2}\{f,\{g,\{h,\{Q, Q\}\}\}\}=0, \quad \forall f, g, h \in \mathcal{M}, \\
((f,(g,(h, R))), R)-\frac{1}{2}(f,(g,(h,(R, R))))=0, \quad \forall f, g, h \in \mathcal{M}^{*} .
\end{gathered}
$$

These conditions should be viewed as conditions on $Q$ and $R$. That there exist solutions may easily be demonstrated in terms of the Darboux coordinates (1.1) on $\mathcal{E}$. We may let $\mathcal{M}$ be the class of functions which only depends on $x^{a}, x_{a}^{*}$. In this case (2.5) is an antibracket on $\mathcal{M}$ which coincide with the antibracket (1.3) on $\mathcal{M}$ if $Q=p_{a} p_{*}^{a}(-1)^{\varepsilon_{a}}$. After quantization this $Q$ is turned into the $\Delta$-operator in the BV-quantization [3]. However, there are many other choices of $\mathcal{M}$. For instance, we may choose $\mathcal{M}$ to be all functions of $p_{a}, p_{*}^{a}$. In this case (2.5) is equal to the antibracket (1.3) on this submanifold $\mathcal{M}$ if $Q=-x^{a} x_{a}^{*}(-1)^{\varepsilon_{a}}$. Similarly for (2.6) we may choose $\mathcal{M}^{*}$ to be all functions on $x^{a}, p_{a}$ in which case (2.6) is the Poisson bracket (1.2) on $\mathcal{M}^{*}$ provided $R=-x_{a}^{*} p_{*}^{a}$, and if $\mathcal{M}^{*}$ is all functions of $x_{a}^{*}, p_{*}^{a}$ then (2.6) is equal to the Poisson bracket (1.2) on this $\mathcal{M}^{*}$ provided $R=-x^{a} p_{a}$. All these choices of $Q$ and $R$ satisfy

$$
\{Q, Q\}=0, \quad(R, R)=0,
$$

which are natural conditions on $Q$ and $R$. From now on we shall always require (2.9). 
The first conditions in (2.7) and (2.8) are naturally restricted to the following conditions

$$
\begin{gathered}
\left\{(f, g)_{Q}, h\right\}=0 \Leftrightarrow(f, g)_{Q} \in \mathcal{M} \quad \forall f, g, h \in \mathcal{M}, \\
\left(\{f, g\}_{R}, h\right)=0 \Leftrightarrow\{f, g\}_{R} \in \mathcal{M}^{*} \quad \forall f, g, h \in \mathcal{M}^{*} .
\end{gathered}
$$

These conditions make the brackets (2.5) and (2.6) closed on the submanifolds $\mathcal{M}$ and $\mathcal{M}^{*}$ respectively.

That the formulas (2.1)-(2.2) or (2.5)-(2.6) may yield arbitrary antibrackets and Poisson brackets may be seen as follows: Let $\mathcal{E}$ be a symplectic manifold with canonical coordinates $z^{A}, p_{A}, A=1, \ldots, 2 n, \varepsilon\left(z^{A}\right)=\varepsilon\left(p_{A}\right) \equiv \varepsilon_{A}$, satisfying $\left\{z^{A}, p_{B}\right\}=\delta_{B}^{A}$. Choose $\mathcal{M}$ to be all functions of $z^{A}$ and

$$
Q=-\frac{1}{2} p_{A} E^{A B}(z) p_{B}(-1)^{\varepsilon_{B}} .
$$

The formula (2.1) or (2.5) yields then the general antibracket (B.7). Note that a nonzero odd $Q$ in (2.12) requires the first equalities in (B.8) and that $\{Q, Q\}=0$ yields the Jacobi identities in (B.8) since the first condition in (2.7) is satisfied by (2.12). (The weaker condition in $(2.7)$ requires here $\{Q, Q\}=0$.) The condition $(2.10)$ is valid for any $Q$ which is at most quadratic in $p_{A}$. Similarly if $\mathcal{E}$ is an antisymplectic manifold with the anticanonical coordinates $x^{A}, x_{A}^{*}, A=1, \ldots, 2 n, \varepsilon\left(x^{A}\right) \equiv \varepsilon_{A}, \varepsilon\left(x_{A}^{*}\right)=\varepsilon_{A}+1$, satisfying $\left(x^{A}, x_{B}^{*}\right)=\delta_{B}^{A}$, and if we choose $\mathcal{M}^{*}$ to be all functions of $x^{A}$ and

$$
R=-\frac{1}{2} x_{A}^{*} \omega^{A B}(x) x_{B}^{*},
$$

then the formula (2.2) or (2.6) yields the general Poisson bracket (A.7) on $\mathcal{M}^{*}$. A nonzero even $R$ requires the first properties in (A.8) while $(R, R)=0$ yields the Jacobi identities. The condition in (2.11) is satisfied by any $R$ at most quadratic in $x_{A}^{*}$. This connection to Poisson brackets was used in [10, 11], and the formula (2.6) was previously given in [4, 5, 6] and the corresponding relation in the $S p(2)$ case was given in [12].

Thus, by means of a nondegenerate Poisson bracket/antibracket on $\mathcal{E}$ we may define a nondegenerate antibracket/Poisson bracket on $\mathcal{M} / \mathcal{M}^{*}$ provided $(2.7) /(2.8)$ is valid. The submanifolds $\mathcal{M}$ and $\mathcal{M}^{*}$ have half the size of $\mathcal{E}$. The dimension of $\mathcal{M}$ is $(n, n)$, and the dimension of $\mathcal{M}^{*}$ is $2 n$ with arbitrary distribution of fermions and bosons depending on the choice of $\mathcal{E}$. Note that the coexistence of an antibracket and a Poisson bracket is trivial on these submanifolds: $\mathcal{M}$ is an antisymplectic manifold on which the original Poisson bracket is zero, while $\mathcal{M}^{*}$ is a symplectic manifold on which the original antibracket is zero. Note that if we e.g. calculate the Poisson bracket (2.2) in terms of the antibracket (2.1) then we get a Poisson bracket defined on a submanifold which is only one-fourth of $\mathcal{E}$. Furthermore, this Poisson bracket is completely different from the original one in (2.1).

We end this section with a remark on equation (1.4) in the introduction. In the above constructions of antibrackets and Poisson brackets expressed in terms of each other we may solve condition (1.4) in the following sense: If we have an antibracket on $\mathcal{E}$ and a Poisson bracket on $\mathcal{M}^{*}$ there always exists an odd Hamiltonian $Q_{H}$ on $\mathcal{E}$ such that $\{f, H\}=\left(f, Q_{H}\right)$ for all $f, H \in \mathcal{M}^{*}$ given by $Q_{H}=(R, H)$ (cf.四). Example: Let $\mathcal{M}^{*}$ be all functions of $x^{a}, p_{a}$ and $R=-x_{a}^{*} p_{*}^{a}$, then we have

$$
\{f(x, p), H(x, p)\}=\left(f(x, p), Q_{H}\right), \quad Q_{H}=x_{a}^{*}\left\{x^{a}, H\right\}+p_{*}^{a}\left\{p_{a}, H\right\} .
$$


Similarly, if we have a Poisson bracket on $\mathcal{E}$ and an antibracket on $\mathcal{M}$ there always exists a Hamiltonian $\mathrm{H}$ on $\mathcal{E}$ for every odd Hamiltonian $Q_{H}$ on $\mathcal{M}$ given by $H=\left\{Q, Q_{H}\right\}$. Example: Let $\mathcal{M}$ be all functions of $x^{a}, x_{a}^{*}$ and $Q=p_{a} p_{*}^{a}(-1)^{\varepsilon_{a}}$, then we have

$$
\begin{aligned}
& \left(f\left(x, x^{*}\right), Q_{H}\left(x, x^{*}\right)\right)=\left\{f\left(x, x^{*}\right), H\right\}, \\
& H=p_{a}\left(x^{a}, Q_{H}\left(x, x^{*}\right)\right)+p_{*}^{a}\left(x_{a}^{*}, Q_{H}\left(x, x^{*}\right)\right) .
\end{aligned}
$$

Thus, we always have counterparts to the Hamiltonians. However, these counterparts are defined on an extended manifold.

\section{Generating functions and generalized Poisson brackets and antibrackets.}

The functions $Q$ and $R$ play a crucial role in the construction of an antibracket on $\mathcal{M}$ or a Poisson bracket on $\mathcal{M}^{*}$ in terms of a nondegenerate Poisson bracket or a nondegenerate antibracket on $\mathcal{E}$ as presented in the previous section. $Q$ and $R$ should satisfy $\{Q, Q\}=0$ and $(R, R)=0$. In addition they have to satisfy the conditions (2.10) and (2.11). Here we propose a generalized scheme in which the latter conditions are relaxed. This scheme involves higher antibrackets and higher Poisson brackets whose properties are most easily obtained by means of generating functions of these brackets. (For quantum antibrackets these relations were given in [3, 7].)

\subsection{Generating functions for generalized antibrackets.}

Let $\mathcal{E}$ be a symplectic manifold. We have then a nondegenerate Poisson bracket and an odd function $Q$ on $\mathcal{E}$ satisfying $\{Q, Q\}=0$. Let us first assume that $f_{a}$ are functions on $\mathcal{M}$, i.e. functions satisfying the abelian algebra $\left\{f_{a}, f_{b}\right\}=0$. We may then define the following canonically transformed $Q$ :

$$
Q(\phi) \equiv Q \exp \left\{\overleftarrow{a d} f_{a} \phi^{a}\right\}, \quad \varepsilon\left(f_{a}\right) \equiv \varepsilon_{a}=\varepsilon\left(\phi^{a}\right)
$$

where

$$
g \stackrel{\leftarrow}{a d} f_{a} \equiv\left\{g, f_{a}\right\}
$$

and where $\phi^{a}$ are parameters. Obviously $\left(\partial_{a} \equiv \partial / \partial \phi^{a}\right)$

$$
Q(0)=Q, \quad Q(\phi) \overleftarrow{\partial_{a}}=\left\{Q(\phi), f_{a}\right\}
$$

In terms of $Q(\phi)$ we may then define the following generalized antibrackets for the functions on $\mathcal{M}$ (cf. [13])

$$
\begin{aligned}
& \left(f_{a_{1}}, f_{a_{2}}, \ldots, f_{a_{n}}\right)_{Q(\phi)} \equiv-Q(\phi) \overleftarrow{\partial}_{a_{1}} \overleftarrow{\partial}_{a_{2}} \cdots \overleftarrow{\partial}_{a_{n}}(-1)^{E_{n}}= \\
& =-\left\{\cdots\left\{\left\{Q(\phi), f_{a_{1}}\right\}, f_{a_{2}}\right\}, \cdots, f_{a_{n}}\right\}(-1)^{E_{n}}, \quad E_{n} \equiv \sum_{k=0}^{\left[\frac{n-1}{2}\right]} \varepsilon_{a_{2 k+1}}
\end{aligned}
$$


They satisfy the properties

$$
\begin{aligned}
& \left(\ldots, f_{a}, f_{b}, \ldots\right)_{Q(\phi)}=-(-1)^{\left(\varepsilon_{a}+1\right)\left(\varepsilon_{b}+1\right)}\left(\ldots, f_{b}, f_{a}, \ldots\right)_{Q(\phi)} \\
& \varepsilon\left(\left(f_{a_{1}}, f_{a_{2}}, \ldots, f_{a_{n}}\right)_{Q(\phi)}\right)=\varepsilon_{a_{1}}+\cdots+\varepsilon_{a_{n}}+1 .
\end{aligned}
$$

Furthermore, since (3.1) is a canonically transformed $Q$ we have

$$
\{Q(\phi), Q(\phi)\}=\{Q, Q\}=0,
$$

from which we may derive identities like

$$
\{Q(\phi), Q(\phi)\} \overleftarrow{\partial}_{a} \overleftarrow{\partial}_{b} \overleftarrow{\partial}_{c}(-1)^{\varepsilon_{b}+\left(\varepsilon_{a}+1\right)\left(\varepsilon_{c}+1\right)} \equiv 0
$$

which in turn implies

$$
\begin{aligned}
& \left(f_{a},\left(f_{b}, f_{c}\right)_{Q(\phi)}\right)_{Q(\phi)}(-1)^{\left(\varepsilon_{a}+1\right)\left(\varepsilon_{c}+1\right)}+\left(f_{c},\left(f_{a}, f_{b}\right)_{Q(\phi)}\right)_{Q(\phi)}(-1)^{\left(\varepsilon_{c}+1\right)\left(\varepsilon_{b}+1\right)}+ \\
& +\left(f_{b},\left(f_{c}, f_{a}\right)_{Q(\phi)}\right)_{Q(\phi)}(-1)^{\left(\varepsilon_{b}+1\right)\left(\varepsilon_{a}+1\right)}=\left\{\left\{\left\{\left\{Q(\phi), f_{a}\right\}, f_{b}\right\}, f_{c}\right\}, Q(\phi)\right\}(-1)^{\varepsilon_{a} \varepsilon_{c}} \equiv \\
& \equiv\left\{\left(f_{a}, f_{b}, f_{c}\right)_{Q(\phi)}, Q(\phi)\right\}(-1)^{\left(\varepsilon_{a}+1\right)\left(\varepsilon_{c}+1\right)} .
\end{aligned}
$$

It is easily seen that condition $(2.10)$ is equivalent to the vanishing of the 3-bracket $(n=3)$ in (3.4). Thus, in this case the 2-bracket in (3.4) is the conventional antibracket considered in the previous section. Relation (3.8) is then just the Jacobi identities of the 2-antibracket.

Now if we relax condition (2.10) the formula (3.8) is not quite satisfactory as it stands. The reason is that the antibracket $(n=2)$ in (3.8) is given by the expression (2.5) which only is valid for functions on $\mathcal{M}$. However, if (2.10) is relaxed then $\left(f_{a}, f_{b}\right)_{Q} \notin \mathcal{M}$ and the outer brackets in (3.8) are not the appropriate ones to use. Below we show that remarkably enough the correct expression have the same form except for a factor minus one-half in the last equality.

A better definition of higher antibrackets are obtained if we consider a more general class of functions in the generating function $Q(\phi)$. Let therefore the functions $f_{a}$ in (3.1) from now on be functions satisfying the Lie algebra

$$
\left\{f_{a}, f_{b}\right\}=U_{a b}^{c} f_{c}, \quad U_{a b}^{d} U_{d c}^{f}(-1)^{\varepsilon_{a} \varepsilon_{c}}+\operatorname{cycle}(a, b, c) \equiv 0 .
$$

where the structure coefficients $U_{a b}^{c}$ are constants. These functions obviously live on a larger manifold than $\mathcal{M}$. Instead of $(3.3)$ we have then

$$
Q(\phi) \overleftarrow{\partial}_{a}=\left\{Q(\phi), \lambda_{a}^{b}(\phi) f_{b}(-1)^{\varepsilon_{a}+\varepsilon_{b}}\right\}
$$

where integrability requires the parameter matrices $\lambda_{a}^{b}(\phi)$ to satisfy the Maurer-Cartan equation

$$
\partial_{a} \lambda_{b}^{c}-\partial_{b} \lambda_{a}^{c}(-1)^{\varepsilon_{a} \varepsilon_{b}}=\lambda_{a}^{e} \lambda_{b}^{d} U_{d e}^{c}(-1)^{\varepsilon_{b} \varepsilon_{e}+\varepsilon_{c}+\varepsilon_{d}+\varepsilon_{e}}, \quad \lambda_{a}^{b}(0)=\delta_{a}^{b} .
$$

The explicit solution is

$$
\tilde{\lambda}=\left(1-e^{-X}\right) / X, \quad \tilde{\lambda}_{b}^{a} \equiv \lambda_{b}^{a}(-1)^{\varepsilon_{a}\left(\varepsilon_{b}+1\right)}, \quad X_{b}^{a} \equiv \phi^{c} U_{c b}^{a}(-1)^{\varepsilon_{c}+\varepsilon_{b}\left(\varepsilon_{a}+1\right)},
$$

where $\phi^{b} \lambda_{b}^{a}=\phi^{a}$. Generalized higher antibrackets are then obtained from the definition (3.4). In particular we have

$$
\begin{aligned}
& \left(f_{a}, f_{b}\right)_{Q(\phi)}^{\prime} \equiv-Q(\phi) \overleftarrow{\partial}_{a} \overleftarrow{\partial}_{b}(-1)^{\varepsilon_{a}}= \\
& =-\lambda_{a}^{c} \lambda_{b}^{d}\left\{\left\{Q(\phi), f_{d}\right\}, f_{c}\right\}(-1)^{\varepsilon_{b} \varepsilon_{c}+\varepsilon_{a}}-\left(\partial_{b} \lambda_{a}^{c}\right)\left\{Q(\phi), f_{c}\right\}(-1)^{\varepsilon_{a}\left(\varepsilon_{b}+1\right)}
\end{aligned}
$$


This expression reduces exactly to the general ansatz (2.1) at $\phi^{a}=0$. However, compared to the case in section 2 we have now a complete control over the Jacobi identities since (3.6) is valid leading to identities like (3.7) involving the true violation of the Jacobi identities when $(2.10)$ is relaxed. Eq.(3.7) yields now at $\phi^{a}=0$

$$
\begin{aligned}
& \left(f_{a},\left(f_{b}, f_{c}\right)_{Q}\right)_{Q}(-1)^{\left(\varepsilon_{a}+1\right)\left(\varepsilon_{c}+1\right)}+\left(f_{c},\left(f_{a}, f_{b}\right)_{Q}\right)_{Q}(-1)^{\left(\varepsilon_{c}+1\right)\left(\varepsilon_{b}+1\right)}+ \\
& +\left(f_{b},\left(f_{c}, f_{a}\right)_{Q}\right)_{Q}(-1)^{\left(\varepsilon_{b}+1\right)\left(\varepsilon_{a}+1\right)}=-\frac{1}{2}\left\{\left(f_{a}, f_{b}, f_{c}\right)_{Q}, Q\right\}(-1)^{\left(\varepsilon_{a}+1\right)\left(\varepsilon_{c}+1\right)},
\end{aligned}
$$

where the higher antibracket is given by

$$
\begin{aligned}
& \left.\left(f_{a}, f_{b}, f_{c}\right)_{Q}(-1)^{\left(\varepsilon_{a}+1\right)\left(\varepsilon_{c}+1\right)} \equiv\left(f_{a}, f_{b}, f_{c}\right)_{Q(\phi)}^{\prime}(-1)^{\left(\varepsilon_{a}+1\right)\left(\varepsilon_{c}+1\right)}\right|_{\phi=0}= \\
& =\frac{1}{3}\left(\left\{\left(f_{a}, f_{b}\right)_{Q}, f_{c}\right\}(-1)^{\varepsilon_{c}+\left(\varepsilon_{a}+1\right)\left(\varepsilon_{c}+1\right)}+\operatorname{cycle}(a, b, c)\right)
\end{aligned}
$$

where in turn the antibrackets are given by (2.1). Although this 3-antibracket is defined as in (3.4) it differs from the explicit expression on the right-hand side of (3.4) since all antibrackets now are given by (2.1). In fact, the definition (3.15) as well as the relation (3.14) are valid for arbitrary dynamical functions, i.e. not just of the class (3.9). (Still higher generalized antibrackets may also be expressed in terms of lower ones.) It is remarkable that (3.14) differs from (3.8) only by a factor.

Note that the class of functions satisfying (3.9) also includes all products of such functions: Define $F_{A}$ to be all monomials of $f_{a}$, i.e. $F_{A} \equiv f_{a}, f_{a} f_{b}, f_{a} f_{b} f_{c}, \ldots$ These operators do also satisfy a nonabelian Lie algebra, $\left\{F_{A}, F_{B}\right\}=\mathcal{U}_{A B}^{C} F_{C}$. In accordance with (3.1) we may therefore define

$$
Q(\Phi) \equiv Q \exp \left\{\overleftarrow{a d} F_{A} \Phi^{A}\right\}
$$

where the parameters $\Phi^{A}$ are $\phi^{a}, \phi^{a b}, \phi^{a b c}, \ldots$ Since $F_{A}$ satisfies a nonabelian Lie algebra we have also here integrable equations of the form (3.10) and generalized antibrackets $\left(\partial_{A}=\partial / \partial \Phi^{A}\right)$

$$
\left(F_{A}, F_{B}\right)_{Q(\Phi)}^{\prime} \equiv-Q(\Phi) \overleftarrow{\partial}_{A} \overleftarrow{\partial}_{B}(-1)^{\varepsilon_{A}}, \quad \varepsilon_{A} \equiv \varepsilon\left(F_{A}\right)=\varepsilon\left(\Phi^{A}\right)
$$

which again reduces to 2.1) at $\Phi^{A}=0$.

Although we have found a consistent generalized scheme in terms of higher antibrackets it should be noted that these generalized antibrackets do not in general satisfy Leibniz' rule (B.5). The violation is given by (2.3). For the class of functions satisfying the Lie algebra (3.9) we have in particular

$$
\begin{aligned}
& \left(f_{a} f_{b}, f_{c}\right)_{Q}-f_{a}\left(f_{b}, f_{c}\right)_{Q}-\left(f_{a}, f_{c}\right)_{Q} f_{b}(-1)^{\varepsilon_{b}\left(\varepsilon_{c}+1\right)}= \\
& =\frac{1}{2}\left(\left\{f_{a}, Q\right\} U_{b c}^{d}(-1)^{\varepsilon_{b}}+\left\{f_{b}, Q\right\} U_{a c}^{d}(-1)^{\varepsilon_{a}\left(\varepsilon_{b}+1\right)}\right) f_{d},
\end{aligned}
$$

which in the case when $f_{a}$ are interpreted as constraint variables may be viewed as a weak violation since the right-hand side vanishes on the constraint surface, $f_{a}=0$. One may notice that if a class of functions satisfy Leibniz' rule for the 2-bracket it is satisfied for all higher antibrackets as well. 


\subsection{Generating functions for generalized Poisson brackets.}

There exists a completely dual construction when $\mathcal{E}$ is an antisymplectic manifold. In this case we have a nondegenerate antibracket and an even function $R$ on $\mathcal{E}$ satisfying $(R, R)=0$. Let to start with $f_{a}$ be functions on $\mathcal{M}^{*}$ satisfying $\left(f_{a}, f_{b}\right)=0$. We may then define the following anticanonically transformed $R$ :

$$
R(\phi) \equiv R \exp \left\{\overleftarrow{A d} f_{a} \phi^{a}\right\}, \quad \varepsilon\left(f_{a}\right) \equiv \varepsilon_{a}, \quad \varepsilon\left(\phi^{a}\right)=\varepsilon_{a}+1
$$

where

$$
g \overleftarrow{A d} f_{a} \equiv\left(g, f_{a}\right)
$$

and where $\phi^{a}$ are parameters. Obviously

$$
R(0)=R, \quad R(\phi) \overleftarrow{\partial_{a}}=\left(R(\phi), f_{a}\right)
$$

In terms of $R(\phi)$ we define the following higher Poisson brackets for functions on $\mathcal{M}^{*}$

$$
\begin{aligned}
& \left\{f_{a_{1}}, f_{a_{2}}, \ldots, f_{a_{n}}\right\}_{R(\phi)} \equiv-R(\phi) \overleftarrow{\partial}_{a_{1}} \overleftarrow{\partial}_{a_{2}} \cdots \overleftarrow{\partial}_{a_{n}}(-1)^{E_{n}}= \\
& =-\left(\cdots\left(\left(R(\phi), f_{a_{1}}\right), f_{a_{2}}\right), \cdots, f_{a_{n}}\right)(-1)^{E_{n}}, \quad E_{n} \equiv \sum_{k=0}^{\left[\frac{n-1}{2}\right]}\left(\varepsilon_{a_{2 k+1}}+1\right)
\end{aligned}
$$

Note the properties

$$
\begin{aligned}
& \left\{\ldots, f_{a}, f_{b}, \ldots\right\}_{R(\phi)}=-(-1)^{\varepsilon_{a} \varepsilon_{b}}\left\{\ldots, f_{b}, f_{a}, \ldots\right\}_{R(\phi)}, \\
& \varepsilon\left(\left\{f_{a_{1}}, f_{a_{2}}, \ldots, f_{a_{n}}\right\}_{R(\phi)}\right)=\varepsilon_{a_{1}}+\cdots+\varepsilon_{a_{n}}+n .
\end{aligned}
$$

To our knowledge these higher Poisson brackets are of a new kind. Nambu once introduced similar higher Poisson brackets [14] but they were not defined as in (3.22).

Since (3.19) is an anticanonically transformed $R$ we have

$$
(R(\phi), R(\phi))=(R, R)=0,
$$

from which we may derive identities like

$$
(R(\phi), R(\phi)) \overleftarrow{\partial}_{a} \overleftarrow{\partial}_{b} \overleftarrow{\partial}_{c}(-1)^{\varepsilon_{b}+\varepsilon_{a} \varepsilon_{c}} \equiv 0
$$

which in turns implies

$$
\begin{aligned}
& \left\{f_{a},\left\{f_{b}, f_{c}\right\}_{R(\phi)}\right\}_{R(\phi)}(-1)^{\varepsilon_{a} \varepsilon_{c}}+\left\{f_{c},\left\{f_{a}, f_{b}\right\}_{R(\phi)}\right\}_{R(\phi)}(-1)^{\varepsilon_{c} \varepsilon_{b}}+ \\
& +\left\{f_{b},\left\{f_{c}, f_{a}\right\}_{R(\phi)}\right\}_{R(\phi)}(-1)^{\varepsilon_{b} \varepsilon_{a}}=\left(\left(\left(\left(R(\phi), f_{a}\right), f_{b}\right), f_{c}\right), R(\phi)\right)(-1)^{\left(\varepsilon_{a}+1\right)\left(\varepsilon_{c}+1\right)} \equiv \\
& \equiv\left(\left\{f_{a}, f_{b}, f_{c}\right\}_{R(\phi)}, R(\phi)\right)(-1)^{\varepsilon_{a} \varepsilon_{c}} .
\end{aligned}
$$

It is easily seen that condition (2.11) is equivalent to the vanishing of the 3-bracket $(n=3)$ in $(3.22)$. Thus, in this case the 2-bracket in (3.22) is the conventional Poisson bracket considered in the previous section. Relation (3.26) is then just the Jacobi identities of the Poisson bracket. 
Similarly to the case in the previous section formula $(3.26)$ is not quite satisfactory if we relax condition (2.11) since the Poisson bracket $(n=2)$ in (3.26) is given by (2.6) which is valid only for functions belonging to $\mathcal{M}^{*}$. However, if (2.11) is relaxed then $\left(f_{a}, f_{b}\right)_{Q} \notin \mathcal{M}$ and the outer brackets in (3.26) are not the appropriate ones. Below it it is shown that the correct expression have the same form except for a factor one-half in the last equality.

Replace the functions in the generating function $R(\phi)$ by functions $f_{a}, \varepsilon\left(f_{a}\right)=\varepsilon_{a}$, satisfying the antibracket Lie algebra

$$
\left(f_{a}, f_{b}\right)=U_{a b}^{c} f_{c}, \quad U_{a b}^{d} U_{d c}^{f}(-1)^{\left(\varepsilon_{a}+1\right)\left(\varepsilon_{c}+1\right)}+\operatorname{cycle}(a, b, c) \equiv 0 .
$$

Note that the structure coefficients here are different from those in (3.9) due to the oddness of the antibracket $\left(\varepsilon\left(U_{a b}^{c}\right)=\varepsilon_{a}+\varepsilon_{b}+\varepsilon_{c}+1\right)$. Instead of (3.21) we have

$$
R(\phi) \overleftarrow{\partial}_{a}=\left(R(\phi), \lambda_{a}^{b}(\phi) f_{b}(-1)^{\varepsilon_{a}+\varepsilon_{b}}\right), \quad \varepsilon\left(\lambda_{a}^{b}\right)=\varepsilon_{a}+\varepsilon_{b}
$$

where integrability requires the parameter matrices $\lambda_{a}^{b}(\phi)\left(\lambda_{a}^{b}(0)=\delta_{a}^{b}\right)$ to satisfy the following Maurer-Cartan equation which is of a new type (cf.(3.11))

$$
\partial_{a} \lambda_{b}^{c}+\partial_{b} \lambda_{a}^{c}(-1)^{\varepsilon_{a} \varepsilon_{b}}=(-1)^{\varepsilon_{a}} \lambda_{a}^{e} \lambda_{b}^{d}(-1)^{\left(\varepsilon_{b}+1\right)\left(\varepsilon_{e}+1\right)} U_{d e}^{c}(-1)^{\varepsilon_{c}+\varepsilon_{d}+\varepsilon_{e}} .
$$

The explicit solution is

$$
\tilde{\lambda}=\left(1-e^{-X}\right) / X, \quad \tilde{\lambda}_{b}^{a} \equiv \lambda_{b}^{a}(-1)^{\varepsilon_{a}\left(\varepsilon_{b}+1\right)}, \quad X_{b}^{a} \equiv \phi^{c} U_{c b}^{a}(-1)^{\varepsilon_{b}\left(\varepsilon_{a}+1\right)},
$$

where now $\phi^{b} \lambda_{b}^{a}(-1)^{\varepsilon_{b}}=\phi^{a}(-1)^{\varepsilon_{a}}$. Note that we have here $\varepsilon\left(\phi^{a}\right)=\varepsilon_{a}+1$. Generalized higher Poisson brackets are defined by (3.22). In particular we have therefore

$$
\begin{aligned}
& \left\{f_{a}, f_{b}\right\}_{R(\phi)}^{\prime} \equiv R(\phi) \overleftarrow{\partial}_{a} \overleftarrow{\partial}_{b}(-1)^{\varepsilon_{a}}= \\
& =\lambda_{a}^{c} \lambda_{b}^{d}\left(\left(R(\phi), f_{d}\right), f_{c}\right)(-1)^{\left(\varepsilon_{b}+1\right)\left(\varepsilon_{c}+1\right)+\varepsilon_{a}}-\left(\partial_{b} \lambda_{a}^{c}\right)\left(R(\phi), f_{c}\right)(-1)^{\varepsilon_{a} \varepsilon_{b}}
\end{aligned}
$$

This expression reduces exactly to the general ansatz $(2.2)$ at $\phi^{a}=0$, and again we have a complete control over the Jacobi identities since (3.24) is valid leading to identities like (3.25) now involving the violation of the true Jacobi identities. We have explicitly

$$
\begin{aligned}
& \left\{f_{a},\left\{f_{b}, f_{c}\right\}_{R}\right\}_{R}(-1)^{\varepsilon_{a} \varepsilon_{c}}+\left\{f_{c},\left\{f_{a}, f_{b}\right\}_{R}\right\}_{R}(-1)^{\varepsilon_{c} \varepsilon_{b}}+ \\
& +\left\{f_{b},\left\{f_{c}, f_{a}\right\}_{R}\right\}_{R}(-1)^{\varepsilon_{b} \varepsilon_{a}}=\frac{1}{2}\left(\left\{f_{a}, f_{b}, f_{c}\right\}_{R}, R\right)(-1)^{\varepsilon_{a} \varepsilon_{c}},
\end{aligned}
$$

where the higher Poisson bracket is given by

$$
\begin{aligned}
& \left.\left\{f_{a}, f_{b}, f_{c}\right\}_{R}(-1)^{\varepsilon_{a} \varepsilon_{c}} \equiv\left\{f_{a}, f_{b}, f_{c}\right\}_{R(\phi)}^{\prime}(-1)^{\varepsilon_{a} \varepsilon_{c}}\right|_{\phi=0}= \\
& =\frac{1}{3}\left(\left(\left\{f_{a}, f_{b}\right\}_{R}, f_{c}\right)(-1)^{\varepsilon_{c}+\varepsilon_{a} \varepsilon_{c}}+\operatorname{cycle}(a, b, c)\right),
\end{aligned}
$$

where in turn the Poisson brackets are given by (2.2). Note that (3.32) differs from (3.8) only by a factor. Also here the class of functions satisfying (3.27) includes all products of such functions.

Instead of Leibniz' rule (A.5) we have from (2.4)

$$
\begin{aligned}
& \left\{f_{a} f_{b}, f_{c}\right\}_{R}-f_{a}\left\{f_{b}, f_{c}\right\}_{R}-\left\{f_{a}, f_{c}\right\}_{R} f_{b}(-1)^{\varepsilon_{b} \varepsilon_{c}}= \\
& =-\frac{1}{2}\left(\left(R, f_{a}\right) U_{b c}^{d}(-1)^{\varepsilon_{b}+\varepsilon_{c}}+\left(R, f_{b}\right) U_{a c}^{d}(-1)^{\left(\varepsilon_{a}+1\right)\left(\varepsilon_{b}+1\right)}\right) f_{d},
\end{aligned}
$$


which in the case when $f_{a}$ are interpreted as constraint variables may be viewed as a weak violation of Leibniz' rule since it vanishes on the constraint surface, $f_{a}=0$. One may notice that if a class of functions satisfy Leibniz' rule for the Poisson bracket it is satisfied for all higher Poisson brackets as well.

\subsection{The most general brackets}

If we consider the brackets (2.1) and (2.2) on the whole of the original manifold $\mathcal{E}$ and require $Q$ and $R$ to satisfy (2.9) then we have a consistent scheme of generalized brackets involving a tower of higher brackets on $\mathcal{E}$. These higher brackets are defined by (3.15) etc and (3.33) etc which may be extracted from the generating functions of the higher brackets for functions satisfying Lie algebra relations in the original bracket. The Jacobi identities of (2.1) and (2.2) are violated by the expressions (3.14) with (3.15) and (3.32) with (3.33), which remarkably enough are valid for arbitrary functions on $\mathcal{E}$. The corresponding relations for the higher brackets of functions satisfying Lie algebra relations, which are obtained by identities like (3.7) and (3.25), should also be valid for arbitrary functions. However, note that the brackets (2.1) and (2.2) violate Leibniz' rule by the expressions (2.3) and (2.4).

\section{Generalized Maurer-Cartan equations}

By means of generating functions of brackets of functions satisfying a Lie algebra in the original brackets we were led to the most general definition of generalized antibrackets and Poisson brackets in terms of higher brackets at the end of the last section. Although there are no generating functions for such brackets involving arbitrary functions on $\mathcal{E}$ there is a further generalization of generating functions which generate the above brackets for functions in arbitrary involutions in the original bracket. This generalization involves a lot of interesting features like a new type of master equation encoding new generalized MaurerCartan equations. This is presented below. (For quantum antibrackets this construction was given in [0].)

\subsection{Functions in arbitrary involutions in a Poisson bracket sense.}

Let $\mathcal{E}$ be a symplectic manifold and let $f_{a}$ be functions on $\mathcal{E}$ satisfying the Poisson algebra

$$
\left\{f_{a}, f_{b}\right\}=U_{a b}^{c} f_{c}, \quad \varepsilon\left(f_{a}\right) \equiv \varepsilon_{a},
$$

where the structure coefficients $U_{a b}^{c}$ now may be arbitrary dynamical functions. This case is most efficiently treated if we extend the original phase space $\mathcal{E}$ by the ghost variables $\mathcal{C}^{a}, \mathcal{P}_{a}, \varepsilon\left(\mathcal{C}^{a}\right)=\varepsilon\left(\mathcal{P}_{a}\right)=\varepsilon_{a}+1$, satisfying $\left\{\mathcal{C}^{a}, \mathcal{P}_{b}\right\}=\delta_{b}^{a}$. We may then always define an odd nilpotent function $\Omega$ with ghost number one according to the prescription

$$
\begin{aligned}
& \Omega=\mathcal{C}^{a} f_{a}+\ldots, \quad\{\Omega, \Omega\}=0, \\
& \{G, \Omega\}=\Omega, \quad G \equiv \mathcal{C}^{a} \mathcal{P}_{a}(-1)^{\varepsilon_{a}},
\end{aligned}
$$

where $G$ is the ghost charge and where the additional terms in $\Omega$ depends on $\mathcal{P}_{a}$ (see [15]). ( $\Omega$ is the BFV-BRST charge.) 
Instead of the explicit form (3.1) of the generating function $Q(\phi)$ used in the previous sections, we define here $Q(\phi)$ by the differential equation

$$
Q(\phi) \overleftarrow{\partial_{a}}=\left\{Q(\phi), Y_{a}(\phi)\right\}, \quad Q(0) \equiv Q
$$

which is similar to (3.10). The connections $Y_{a}(\phi), \varepsilon\left(Y_{a}\right)=\varepsilon_{a}$, satisfy the integrability conditions (zero curvature condition)

$$
Y_{a} \overleftarrow{\partial_{b}}-Y_{b} \overleftarrow{\partial_{a}}(-1)^{\varepsilon_{a} \varepsilon_{b}}=\left\{Y_{a}, Y_{b}\right\}
$$

which in turn are integrable. Note that (4.3) implies

$$
\{Q(\phi), Q(\phi)\} \overleftarrow{\partial_{a}}=-\left\{Y_{a}(\phi),\{Q(\phi), Q(\phi)\}\right\}
$$

Thus, the boundary condition $\{Q(0), Q(0)\}=0$ implies $\{Q(\phi), Q(\phi)\}=0$, which shows that $Q \rightarrow Q(\phi)$ is a canonical transformation.

Generalized antibrackets may be defined by (3.4) with $f_{a}$ replaced by $Y_{a}$. In particular we have

$$
\begin{aligned}
& \left(Y_{a}(\phi), Y_{b}(\phi)\right)_{Q(\phi)}^{\prime} \equiv-Q(\phi) \overleftarrow{\partial_{a}} \overleftarrow{\partial_{b}}(-1)^{\varepsilon_{a}}= \\
& =\frac{1}{2}\left(\left\{Y_{a},\left\{Q(\phi), Y_{b}\right\}\right\}-\left\{Y_{b},\left\{Q(\phi), Y_{a}\right\}(-1)^{\left(\varepsilon_{a}+1\right)\left(\varepsilon_{b}+1\right)}\right)-\right. \\
& -\frac{1}{2}\left\{Q(\phi), Y_{a} \overleftarrow{\partial_{b}}+Y_{b} \overleftarrow{\partial_{a}}(-1)^{\varepsilon_{a} \varepsilon_{b}}\right\}(-1)^{\varepsilon_{a}}
\end{aligned}
$$

which should coincide with (2.1) at $\phi^{a}=0$. We expect therefore the last line in (4.6) to vanish at $\phi^{a}=0$. Indeed, this is the case for the first rank quasigroup theories treated below. For the next higher antibracket we find

$$
\begin{aligned}
& \left(Y_{a}(\phi), Y_{b}(\phi), Y_{c}(\phi)\right)_{Q(\phi)}^{\prime}(-1)^{\left(\varepsilon_{a}+1\right)\left(\varepsilon_{c}+1\right)} \equiv Q(\phi) \overleftarrow{\partial_{a}} \overleftarrow{\partial_{b}} \overleftarrow{\partial_{c}}(-1)^{\varepsilon_{a} \varepsilon_{c}}= \\
& =\frac{1}{3}\left(\left\{\left(Y_{a}, Y_{b}\right)_{Q}^{\prime}, Y_{c}\right\}(-1)^{\varepsilon_{c}+\left(\varepsilon_{a}+1\right)\left(\varepsilon_{c}+1\right)}+\operatorname{cycle}(a, b, c)\right)+ \\
& +\frac{1}{3}\left(\left\{\left\{Q, Y_{a}\right\}, Y_{b} \overleftarrow{\partial_{c}}+Y_{c} \overleftarrow{\partial_{b}}(-1)^{\varepsilon_{b} \varepsilon_{c}}\right\}(-1)^{\varepsilon_{a} \varepsilon_{c}}+\operatorname{cycle}(a, b, c)\right)+ \\
& +\frac{1}{6}\left(\left\{Q,\left(Y_{a} \overleftarrow{\partial_{b}}+Y_{b} \overleftarrow{\partial_{a}}(-1)^{\varepsilon_{a} \varepsilon_{b}}\right) \overleftarrow{\partial_{c}}\right\}(-1)^{\varepsilon_{a} \varepsilon_{c}}+\operatorname{cycle}(a, b, c)\right)
\end{aligned}
$$

which differ from (3.32) by similar terms as (4.6) differ from (2.1). Again we expect these deviations to vanish at $\phi^{a}=0$. The same situation is expected to occur for all higher antibrackets, i.e. they are expected to coincide with the generalized higher antibrackets of section 4 at $\phi^{a}=0$. As we already have mentioned in the previous section the generalized brackets above do not satisfy Leibniz' rule. However, for the functions $Y_{a}$ we have from (2.3)

$$
\begin{aligned}
& \left(Y_{a} Y_{b}, Y_{c}\right)_{Q}-Y_{a}\left(Y_{b}, Y_{c}\right)_{Q}-\left(Y_{a}, Y_{c}\right)_{Q} Y_{b}(-1)^{\varepsilon_{b}\left(\varepsilon_{c}+1\right)}= \\
& =\frac{1}{2}\left\{Y_{a}, Q\right\}\left\{Y_{b}, Y_{c}\right\}(-1)^{\varepsilon_{b}}+\frac{1}{2}\left\{Y_{a}, Y_{c}\right\}\left\{Y_{b}, Q\right\}(-1)^{\varepsilon_{c}\left(\varepsilon_{b}+1\right)},
\end{aligned}
$$

where the right-hand side vanishes on the hypersurface determined by $\mathcal{P}_{a}=0$ and $\theta_{a}=0$. 
Following [7] we propose now the following manifestly BRST invariant expression for the connections $Y_{a}$ in 4.3

$$
Y_{a}(\phi) \equiv\left\{\Omega, \Omega_{a}(\phi)\right\}, \quad \varepsilon\left(\Omega_{a}\right)=\varepsilon_{a}+1 .
$$

The integrability conditions (4.4) become then in terms of $\Omega_{a}$

$$
\Omega_{a} \overleftarrow{\partial_{b}}-\Omega_{b} \overleftarrow{\partial_{a}}(-1)^{\varepsilon_{a} \varepsilon_{b}}=\left(\Omega_{a}, \Omega_{b}\right)_{\Omega}-\frac{1}{2}\left\{\Omega_{a b}, \Omega\right\}
$$

where we have introduced the $\Omega$-antibracket defined in accordance with (2.1), i.e.

$$
(A, B)_{\Omega} \equiv \frac{1}{2}\left(\{A,\{\Omega, B\}\}-\{B,\{\Omega, A\}\}(-1)^{\left(\varepsilon_{A}+1\right)\left(\varepsilon_{B}+1\right)}\right) .
$$

We expect $Y_{a}(\phi)$ to be of the form

$$
Y_{a}(\phi)=\lambda_{a}^{b}(\phi) f_{b}(-1)^{\varepsilon_{a}+\varepsilon_{b}}+\{\text { possible ghost dependent terms }\}, \lambda_{a}^{b}(0)=\delta_{a}^{b},
$$

which makes (4.3) similar to (3.10), although $\lambda_{a}^{b}(\phi)$ may be dynamical functions here. The definition (4.9) requires then $\Omega_{a}$ to be of the form

$$
\Omega_{a}(\phi)=\lambda_{a}^{b}(\phi) \mathcal{P}_{b}+\{\text { possible ghost dependent terms }\} .
$$

This means in turn that (4.10) are generalized Maurer-Cartan equations for $\lambda_{a}^{b}(\phi), \varepsilon\left(\lambda_{a}^{b}\right)=$ $\varepsilon_{a}+\varepsilon_{b}$. The integrability conditions of (4.10) involve first derivatives of $\Omega_{a b}$, a third order antibracket, and a new function $\Omega_{a b c}$. The subsequent integrability conditions involve still higher $\Omega$-antibrackets and functions $\Omega_{a b c \ldots}$ with still more indices. However, these integrability conditions are only implicit equations for the functions $\Omega_{a b c \ldots}$.

Following the treatment in [7] we propose below in (4.17) one single master equation involving only two basic dynamical functions which determines $\Omega_{a}, \Omega_{a b}$, etc. One of these dynamical functions is an extended BFV-BRST charge $\Delta$ defined by

$$
\begin{aligned}
& \Delta \equiv \Omega+\eta^{a} \pi_{a}(-1)^{\varepsilon_{a}}, \quad\{\Delta, \Delta\}=0, \\
& \left\{\phi^{a}, \pi_{b}\right\}=\delta_{b}^{a}, \quad \varepsilon\left(\eta^{a}\right)=\varepsilon_{a}+1, \quad \varepsilon\left(\pi_{a}\right)=\varepsilon_{a},
\end{aligned}
$$

where we have introduced the canonical conjugate variables $\pi_{a}$ to $\phi^{a}$, now turned dynamical, and the new ghost variables $\eta^{a}$, to be treated as parameters. The other basic function is an even, extended ghost charge defined by

$$
\begin{aligned}
& S(\phi, \eta) \equiv G+\eta^{a} \Omega_{a}(\phi)+\frac{1}{2} \eta^{b} \eta^{a} \Omega_{a b}(\phi)(-1)^{\varepsilon_{b}}+ \\
& +\frac{1}{6} \eta^{c} \eta^{b} \eta^{a} \Omega_{a b c}(\phi)(-1)^{\varepsilon_{b}+\varepsilon_{a} \varepsilon_{c}}+\cdots \\
& \cdots+\frac{1}{n !} \eta^{a_{n}} \cdots \eta^{a_{1}} \Omega_{a_{1} \cdots a_{n}}(\phi)(-1)^{\left(\varepsilon_{a_{2}}+\ldots+\varepsilon_{a_{n-1}}+\varepsilon_{a_{1}} \varepsilon_{a_{n}}\right)}+\cdots
\end{aligned}
$$

where $G$ is the ghost charge in (4.2). $\Omega_{a_{1} \cdots a_{n}}(\phi)$ are dynamical functions with the properties

$$
\varepsilon\left(\Omega_{a_{1} \cdots a_{n}}(\phi)\right)=\varepsilon_{a_{1}}+\cdots+\varepsilon_{a_{n}}+n, \quad\left\{G, \Omega_{a_{1} \cdots a_{n}}\right\}=-n \Omega_{a_{1} \cdots a_{n}} .
$$

Thus, $\Omega_{a_{1} \cdots a_{n}}$ has ghost number $-n$, which means that if we assign ghost number one to $\eta^{a}$ then $\Delta$ has ghost number one and $S$ has ghost number zero. We propose now that the 
functions $\Omega_{a_{1} \cdots a_{n}}(\phi)$ are related to the ones in the integrability conditions above and that they are determined by the master equation

$$
(S, S)_{\Delta}=\{\Delta, S\}
$$

where the antibracket is defined by (2.1), i.e. we have

$$
(S, S)_{\Delta} \equiv\{\{S, \Delta\}, S\}
$$

Consistency requires $\{\Delta, S\}$ to be nilpotent in a Poisson bracket sense since

$$
\left\{\Delta,(S, S)_{\Delta}\right\}=0 \quad \Leftrightarrow \quad\{\{\Delta, S\},\{\Delta, S\}\}=0 .
$$

The explicit form of $\{S, \Delta\}(=-\{\Delta, S\})$ is

$$
\begin{aligned}
& \{S, \Delta\}=\Omega+\eta^{a}\left\{\Omega_{a}, \Omega\right\}+\eta^{b} \eta^{a} \Omega_{a} \overleftarrow{\partial_{b}}(-1)^{\varepsilon_{b}}+\frac{1}{2} \eta^{b} \eta^{a}\left\{\Omega_{a b}, \Omega\right\}(-1)^{\varepsilon_{b}}+ \\
& +\frac{1}{2} \eta^{c} \eta^{b} \eta^{a} \Omega_{a b} \overleftarrow{\partial_{c}}(-1)^{\varepsilon_{b}+\varepsilon_{c}}+\frac{1}{6} \eta^{c} \eta^{b} \eta^{a}\left\{\Omega_{a b c}, \Omega\right\}(-1)^{\varepsilon_{b}+\varepsilon_{a} \varepsilon_{c}}+O\left(\eta^{4}\right)
\end{aligned}
$$

Note that (4.17) may be written as $\{S,\{S, \Delta\}\}=\{S, \Delta\}$ which when compared with $\{G, \Omega\}=\Omega$ is consistent with $S$ as an extended ghost charge and with $\{S, \Delta\}$ as an extended BRST charge.

To zeroth and first order in $\eta^{a}$ the master equation (4.17) is satisfied identically. However, to second order in $\eta^{a}$ it yields exactly the integrability conditions (4.10). At the third order in $\eta^{a}$ it yields

$$
\begin{aligned}
& \Omega_{b c} \overleftarrow{\partial_{a}}(-1)^{\left(\varepsilon_{b}+1\right) \varepsilon_{a}}+\frac{1}{2}\left(\Omega_{a}, \Omega_{b c}\right)_{\Omega}(-1)^{\varepsilon_{a} \varepsilon_{c}}+\operatorname{cycle}(a, b, c)= \\
& =-\left(\Omega_{a}, \Omega_{b}, \Omega_{c}\right)_{\Omega}(-1)^{\varepsilon_{a} \varepsilon_{c}}-\frac{2}{3}\left\{\Omega_{a b c}^{\prime}, \Omega\right\} \\
& \Omega_{a b c}^{\prime} \equiv \Omega_{a b c}-\frac{1}{8}\left(\left\{\Omega_{a b}, \Omega_{c}\right\}(-1)^{\varepsilon_{a} \varepsilon_{c}}+\operatorname{cycle}(a, b, c)\right),
\end{aligned}
$$

where the higher $\Omega$-antibracket is defined in accordance with (3.14). The above equations are indeed consistent with the integrability conditions of (4.10). At higher orders in $\eta^{a}$ the master equation (4.17) yields equations involving still higher $\Omega$-antibrackets and functions $\Omega_{a b c . .}$ with still more indices. These equations we expect to be consistent with the integrability conditions of (4.21). This chain of integrability conditions will then terminate as soon as a higher order antibracket is zero. We expect this level to be connected to the rank of the theory, i.e. the highest power of $\mathcal{P}_{a}$ in $\Omega$. For instance, if $\Omega$ is of rank $r$ it would be natural to have $\left(\Omega_{a_{1}}, \Omega_{a_{2}}, \ldots, \Omega_{a_{n}}\right)_{\Omega}=0$ for $n>r+1$ in which case $\Omega_{a_{1} a_{2} a_{3} \cdots a_{n}}=0$ for $n>r$ (see below).

As an illustration of our formulas we consider now functions $f_{a}$ forming a quasigroup rank one theory. In this case we have (cf.[0])

$$
\Omega=\mathcal{C}^{a} f_{a}+\frac{1}{2} \mathcal{C}^{b} \mathcal{C}^{a} U_{a b}^{c} \mathcal{P}_{c}(-1)^{\varepsilon_{c}+\varepsilon_{b}} .
$$

where $\{\Omega, \Omega\}=0$ requires $\left\{f_{a}, f_{b}\right\}=U_{a b}^{c} f_{c}$ and

$$
\left(U_{a b}^{d} U_{d c}^{e}+\left\{U_{a b}^{e}, f_{c}\right\}(-1)^{\varepsilon_{c} \varepsilon_{e}}\right)(-1)^{\varepsilon_{a} \varepsilon_{c}}+\operatorname{cycle}(a, b, c) \equiv 0
$$


plus conditions on $\left\{U_{a b}^{c}, U_{d e}^{f}\right\}$. The latter conditions are satisfied if

$$
\left\{U_{a b}^{c}, U_{d e}^{f}\right\}=0, \quad\left\{\left\{f_{d}, U_{a b}^{c}\right\}, U_{e f}^{g}\right\}=0,
$$

which are stronger conditions than what is required by $\{\Omega, \Omega\}=0$. Here $\Omega_{a}$ may be chosen to be

$$
\Omega_{a}(\phi)=\lambda_{a}^{b}(\phi) \mathcal{P}_{b}, \quad \lambda_{a}^{b}(0)=\delta_{a}^{b}
$$

where we assume that

$$
\left\{\lambda_{a}^{b}(\phi), \lambda_{c}^{d}(\phi)\right\}=0, \quad \Leftrightarrow \quad\left\{\Omega_{a}(\phi), \Omega_{b}(\phi)\right\}=0 .
$$

We require now

$$
\left(\Omega_{a}, \Omega_{b}, \Omega_{c}\right)_{\Omega}=0 \quad \Leftrightarrow \quad\left\{\left(\Omega_{a}, \Omega_{b}\right)_{\Omega}, \Omega_{c}\right\}=0,
$$

which allows for the choice $\Omega_{a b}=0$ since (4.27) makes the antibracket $\left(\Omega_{a}, \Omega_{b}\right)_{\Omega}$ satisfy the Jacobi identities, which in turn makes (4.10) integrable without the need of a nontrivial $\Omega_{a b}$. All higher integrability conditions are then identically zero. Condition (4.27) is satisfied if we impose

$$
\left\{\lambda_{a}^{b}(\phi), U_{d e}^{c}\right\}=0, \quad\left\{\lambda_{a}^{b},\left\{\lambda_{c}^{d}, f_{e}\right\}\right\}=0
$$

All the above conditions specify quasigroups [16]. We turn now to eq.(4.10). We find then

$$
\begin{aligned}
& \left(\Omega_{a}, \Omega_{b}\right)_{\Omega}=\left\{\Omega_{a},\left\{\Omega, \Omega_{b}\right\}\right\}=-\lambda_{a}^{f} \lambda_{b}^{e} U_{e f}^{d} \mathcal{P}_{d}(-1)^{\varepsilon_{d}+\varepsilon_{e}+\varepsilon_{f}+\varepsilon_{b} \varepsilon_{f}}+ \\
& +\left(\lambda_{a}^{c}\left\{f_{c}, \lambda_{b}^{d}\right\}-\lambda_{b}^{c}\left\{f_{c}, \lambda_{a}^{d}\right\}(-1)^{\varepsilon_{a} \varepsilon_{b}}\right) \mathcal{P}_{d}(-1)^{\varepsilon_{c}}
\end{aligned}
$$

which implies that eq. 4.10 ) here may be written as

$$
\partial_{a} \tilde{\lambda}_{b}^{c}-\partial_{b} \tilde{\lambda}_{a}^{c}(-1)^{\varepsilon_{a} \varepsilon_{b}}=\tilde{\lambda}_{a}^{e} \tilde{\lambda}_{b}^{d} \tilde{U}_{d e}^{c}(-1)^{\varepsilon_{b} \varepsilon_{e}+\varepsilon_{c}+\varepsilon_{d}+\varepsilon_{e}} .
$$

where $\tilde{\lambda}_{a}^{b} \equiv V \lambda_{a}^{b}$ and $\tilde{U}_{a b}^{c} \equiv V U_{a b}^{c}$, where in turn the differential operator $V$ satisfies the equation

$$
\partial_{a} V=\operatorname{Vad}\left(H_{a}\right), \quad H_{a} \equiv \lambda_{a}^{b} f_{b}(-1)^{\varepsilon_{b}}
$$

where in turn $a d(A) X=\{A, X\}$ for any dynamical function $X$. The integrability condition of $(4.31)$ is

$$
\partial_{a} H_{b}-\partial_{b} H_{a}(-1)^{\varepsilon_{a} \varepsilon_{b}}+\left\{H_{a}, H_{b}\right\}=0
$$

which is equivalent to (4.30).

One may note that

$$
\begin{aligned}
& Y_{a}(\phi)=\left\{\Omega, \Omega_{a}\right\}=\lambda_{a}^{b} f_{b}(-1)^{\varepsilon_{a}+\varepsilon_{b}}+ \\
& +\lambda_{a}^{b} \mathcal{C}^{d} U_{d b}^{c} \mathcal{P}_{c}(-1)^{\varepsilon_{a}+\varepsilon_{c}}+\mathcal{C}^{b}\left\{f_{b}, \lambda_{a}^{c}\right\} \mathcal{P}_{c}
\end{aligned}
$$

If we assume $Q(\phi)$ to have zero Poisson bracket with the last term in (4.33), then (4.3) reduces to (3.10), which was used for quasigroups in [3, []. 


\subsection{Functions in arbitrary involutions in an antibracket sense.}

Let $\mathcal{E}$ be an antisymplectic manifold and let $f_{a}$ be functions on $\mathcal{E}$ satisfying the antibracket algebra

$$
\left(f_{a}, f_{b}\right)=U_{a b}^{c} f_{c}, \quad \varepsilon\left(f_{a}\right) \equiv \varepsilon_{a}, \quad \varepsilon\left(U_{a b}^{c}\right)=\varepsilon_{a}+\varepsilon_{b}+\varepsilon_{c}+1 .
$$

The structure coefficients $U_{a b}^{c}$ are arbitrary dynamical functions and different from the ones in (4.1) due to the oddness of the antibracket. This case is most efficiently treated if we extend the original antisymplectic manifold $\mathcal{E}$ by the ghost and antighost variables $\mathcal{C}^{a}, \mathcal{C}_{a}^{*}, \varepsilon\left(\mathcal{C}^{a}\right)=\varepsilon_{a}, \varepsilon\left(\mathcal{C}_{a}^{*}\right)=\varepsilon_{a}+1$, satisfying $\left(\mathcal{C}^{a}, \mathcal{C}_{b}^{*}\right)=\delta_{b}^{a}$. We may then always define an even function $\Omega$ with ghost number one according to the prescription

$$
\begin{aligned}
& \Omega=\mathcal{C}^{a} f_{a}+\ldots, \quad(\Omega, \Omega)=0 \\
& (G, \Omega)=\Omega, \quad G \equiv-\mathcal{C}^{a} \mathcal{C}_{a}^{*}
\end{aligned}
$$

where $G$ is an odd ghost number function and where the additional terms in $\Omega$ depends on $\mathcal{C}_{a}^{*}$ (see [17]). In analogy with the previous construction we define the generating function $R(\phi)$ by the differential equation

$$
R(\phi) \overleftarrow{\partial_{a}}=\left(R(\phi), Y_{a}(\phi)\right), \quad R(0)=R
$$

where $\phi^{a}, \varepsilon\left(\phi^{a}\right)=\varepsilon_{a}+1$ are parameters and where $Y_{a}(\phi), \varepsilon\left(Y_{a}\right)=\varepsilon_{a}$, satisfies the integrability conditions

$$
Y_{a} \overleftarrow{\partial_{b}}-Y_{b} \overleftarrow{\partial_{a}}(-1)^{\left(\varepsilon_{a}+1\right)\left(\varepsilon_{b}+1\right)}=\left(Y_{a}, Y_{b}\right)
$$

which in turn are integrable. $Y_{a}(\phi)$ is of the form

$$
Y_{a}(\phi)=\lambda_{a}^{b}(\phi) f_{b}(-1)^{\varepsilon_{a}+\varepsilon_{b}}+\{\text { possible ghost dependent terms }\}, \lambda_{a}^{b}(0)=\delta_{a}^{b},
$$

which makes (4.36) similar to (3.28). (However, $\lambda_{a}^{b}(\phi)$ may be dynamical functions here.) Since (4.36) implies

$$
(R(\phi), R(\phi)) \overleftarrow{\partial_{a}}=\left(Y_{a}(\phi),(R(\phi), R(\phi))\right)
$$

the boundary condition $(R(0), R(0))=0$ implies $(R(\phi), R(\phi))=0$ which shows that $R \rightarrow R(\phi)$ is an anticanonical transformation.

The generalized Poisson brackets are defined by (3.22) with $f_{a}$ replaced by $Y_{a}$. In particular we have

$$
\begin{aligned}
& \left\{Y_{a}(\phi), Y_{b}(\phi)\right\}_{R(\phi)}^{\prime} \equiv R(\phi) \overleftarrow{\partial_{a}} \overleftarrow{\partial_{b}}(-1)^{\varepsilon_{a}}= \\
& =\frac{1}{2}\left(\left(Y_{a},\left(R(\phi), Y_{b}\right)\right)-\left(Y_{b},\left(R(\phi), Y_{a}\right)\right)(-1)^{\varepsilon_{a} \varepsilon_{b}}\right)+ \\
& +\frac{1}{2}\left(R(\phi), Y_{a} \overleftarrow{\partial_{b}}+Y_{b} \overleftarrow{\partial_{a}}(-1)^{\left(\varepsilon_{a}+1\right)\left(\varepsilon_{b}+1\right)}\right)(-1)^{\varepsilon_{a}}
\end{aligned}
$$

We expect the last line to vanish at $\phi^{a}=0$ in which case (4.40) coincides with (2.2). Indeed, this is the case for the first rank quasigroup theories treated below. For generalized higher order Poisson brackets we should have a dual situation to the one in (4.7), i.e. we 
expect them to coincide with the generalized higher order Poisson brackets in section 4.2 at $\phi^{a}=0$. Although the above generalized Poisson brackets do not satisfy Leibniz' rule according to (2.4), we have for the functions $Y_{a}$

$$
\begin{aligned}
& \left\{Y_{a} Y_{b}, Y_{c}\right\}_{R}-Y_{a}\left\{Y_{b}, Y_{c}\right\}_{R}-\left\{Y_{a}, Y_{c}\right\}_{R} Y_{b}(-1)^{\varepsilon_{b} \varepsilon_{c}}= \\
& =-\frac{1}{2}\left(R, Y_{a}\right)\left(Y_{b}, Y_{c}\right)(-1)^{\varepsilon_{b}+\varepsilon_{c}}-\frac{1}{2}\left(Y_{a}, Y_{c}\right)\left(R, Y_{b}\right)(-1)^{\varepsilon_{c}\left(\varepsilon_{b}+1\right)},
\end{aligned}
$$

which vanishes at the hypersurface determined by $\mathcal{C}_{a}^{*}=0$ and $\theta_{a}=0$.

Analogously to $(4.9)$ we propose here the following form for the connections $Y_{a}$ in (4.36)

$$
Y_{a}(\phi) \equiv\left(\Omega, \Omega_{a}(\phi)\right), \quad \varepsilon\left(\Omega_{a}\right)=\varepsilon_{a}+1
$$

Comparison with (4.38) requires then $\Omega_{a}$ to be of the form

$$
\Omega_{a}(\phi)=\lambda_{a}^{b}(\phi) \mathcal{C}_{b}^{*}(-1)^{\varepsilon_{b}}+\{\text { possible ghost dependent terms }\} .
$$

The integrability conditions (4.37) become in terms of $\Omega_{a}$

$$
\Omega_{a} \overleftarrow{\partial_{b}}-\Omega_{b} \overleftarrow{\partial_{a}}(-1)^{\left(\varepsilon_{a}+1\right)\left(\varepsilon_{b}+1\right)}=\left\{\Omega_{a}, \Omega_{b}\right\}_{\Omega}-\frac{1}{2}\left(\Omega_{a b}, \Omega\right)
$$

where we have introduced the $\Omega$-bracket which is a Poisson bracket defined in accordance with (2.2), i.e.

$$
\{A, B\}_{\Omega} \equiv \frac{1}{2}\left((A,(\Omega, B))-(B,(\Omega, A))(-1)^{\varepsilon_{A} \varepsilon_{B}}\right) .
$$

The form (4.43) means then that (4.44) is a new kind of generalized Maurer-Cartan equations for $\lambda_{a}^{b}(\phi), \varepsilon\left(\lambda_{a}^{b}\right)=\varepsilon_{a}+\varepsilon_{b}$. It is clear that the integrability conditions of (4.44) will involve higher order Poisson brackets and $\Omega_{a b c \ldots}$ with still more indices.

Also here we propose one single master equation involving only two basic dynamical functions which determines $\Omega_{a}, \Omega_{a b}$, etc. As in section 5.1 we first make $\phi^{a}$ dynamical but now by the introduction of antifields $\phi_{a}^{*}$. Then we introduce new ghost variables $\eta^{a}$, $\varepsilon\left(\eta^{a}\right)=\varepsilon_{a}$, which are to be treated as parameters. By means of $\phi_{a}^{*}$ and $\eta^{a}$ we define then the extended even $\Omega$-function, $\Delta$, defined by

$$
\begin{aligned}
& \Delta \equiv \Omega-\eta^{a} \phi_{a}^{*}(-1)^{\varepsilon_{a}}, \quad(\Delta, \Delta)=0 \\
& \left(\phi^{a}, \phi_{b}^{*}\right)=\delta_{b}^{a}, \quad \varepsilon\left(\eta^{a}\right)=\varepsilon\left(\phi_{a}^{*}\right)=\varepsilon_{a} .
\end{aligned}
$$

In addition we introduce an odd function $S(\phi, \eta)$ defined by

$$
\begin{aligned}
& S(\phi, \eta)=G+\eta^{a} \Omega_{a}(\phi)-\frac{1}{2} \eta^{b} \eta^{a} \Omega_{a b}(\phi)(-1)^{\varepsilon_{b}}+ \\
& +\frac{1}{6} \eta^{c} \eta^{b} \eta^{a} \Omega_{a b c}(\phi)(-1)^{\left(\varepsilon_{a}+\varepsilon_{b}+\varepsilon_{c}+\varepsilon_{a} \varepsilon_{c}\right)}+\cdots \\
& \cdots+\frac{1}{n !} \eta^{a_{n}} \cdots \eta^{a_{1}} \Omega_{a_{1} \cdots a_{n}}(\phi)(-1)^{\left(\varepsilon_{a_{1}}+\varepsilon_{a_{2}}+\ldots+\varepsilon_{a_{n}}+\varepsilon_{a_{1}} \varepsilon_{a_{n}}+n\right)}+\cdots
\end{aligned}
$$

where $G$ is the even ghost number function in (4.35). $\Omega_{a_{1} \cdots a_{n}}(\phi)$ are dynamical functions with the properties

$$
\varepsilon\left(\Omega_{a_{1} \cdots a_{n}}(\phi)\right)=\varepsilon_{a_{1}}+\cdots+\varepsilon_{a_{n}}+1, \quad\left(G, \Omega_{a_{1} \cdots a_{n}}\right)=-n \Omega_{a_{1} \cdots a_{n}},
$$


where the last relation implies that $\Omega_{a_{1} \cdots a_{n}}$ has ghost number $-n$. Thus, if we assign ghost number one to $\eta^{a}$ then $\Delta$ has ghost number one and $S$ has ghost number zero. We propose now that the functions $\Omega_{a_{1} \cdots a_{n}}(\phi)$ are related to the ones in the integrability conditions above and that they are determined by the master equation (cf.(4.17))

$$
\{S, S\}_{\Delta}=(\Delta, S)
$$

where the Poisson bracket is defined by (2.2), i.e. we have

$$
\{S, S\}_{\Delta} \equiv((S, \Delta), S)
$$

Consistency requires $(\Delta, S)$ to satisfy

$$
\left(\Delta,\{S, S\}_{\Delta}\right)=0 \quad \Leftrightarrow \quad((\Delta, S),(\Delta, S))=0 .
$$

The explicit form of $(S, \Delta)(=-(\Delta, S))$ is

$$
\begin{aligned}
& (S, \Delta)=\Omega+\eta^{a}\left(\Omega_{a}, \Omega\right)-\eta^{b} \eta^{a} \Omega_{a} \overleftarrow{\partial_{b}}(-1)^{\varepsilon_{b}}-\frac{1}{2} \eta^{b} \eta^{a}\left(\Omega_{a b}, \Omega\right)(-1)^{\varepsilon_{b}}+ \\
& +\frac{1}{2} \eta^{c} \eta^{b} \eta^{a} \Omega_{a b} \overleftarrow{\partial_{c}}(-1)^{\varepsilon_{b}+\varepsilon_{c}}+\frac{1}{6} \eta^{c} \eta^{b} \eta^{a}\left(\Omega_{a b c}, \Omega\right)(-1)^{\varepsilon_{a}+\varepsilon_{b}+\varepsilon_{c}+\varepsilon_{a} \varepsilon_{c}}+O\left(\eta^{4}\right)
\end{aligned}
$$

Also here (4.49) is of the form $(G, \Omega)=\Omega$ with $G$ given by $S$ and $\Omega$ by $(S, \Delta)$ which shows that $S$ may be viewed as an extended ghost charge.

To zeroth and first order in $\eta^{a}$ eq.(4.49) is satisfied identically. However, to second order in $\eta^{a}$ it yields (4.44). The consistency condition (4.51) yields on the other hand exactly (4.37) when (4.42) is used to second order in $\eta^{a}$. At the third order in $\eta^{a}$ the master equation (4.49) yields

$$
\begin{aligned}
& \Omega_{b c} \overleftarrow{\partial_{a}}(-1)^{\varepsilon_{b}\left(\varepsilon_{a}+1\right)}+\frac{1}{2}\left\{\Omega_{a}, \Omega_{b c}\right\}_{\Omega}(-1)^{\left(\varepsilon_{a}+1\right)\left(\varepsilon_{c}+1\right)}+\operatorname{cycle}(a, b, c)= \\
& =\left\{\Omega_{a}, \Omega_{b}, \Omega_{c}\right\}_{\Omega}(-1)^{\left(\varepsilon_{a}+1\right)\left(\varepsilon_{c}+1\right)}-\frac{2}{3}\left\{\Omega_{a b c}^{\prime}, \Omega\right\} \\
& \Omega_{a b c}^{\prime} \equiv \Omega_{a b c}+\frac{1}{8}\left(\left(\Omega_{a b}, \Omega_{c}\right)(-1)^{\left(\varepsilon_{a}+1\right)\left(\varepsilon_{c}+1\right)}+\operatorname{cycle}(a, b, c)\right),
\end{aligned}
$$

where the higher $\Omega$-bracket is defined in accordance with (3.32). The above equations are also integrability conditions of (4.44). At higher orders in $\eta^{a}$ the master equation (4.49) yields equations involving still higher $\Omega$-brackets and functions $\Omega_{a b c \ldots .}$ with still more indices. We expect these equations to coincide with the integrability conditions of (4.53). This chain of integrability conditions will terminate as soon as a higher order Poisson bracket is zero. Also here this level should be connected to the rank of the theory, i.e. the highest power of $\mathcal{C}_{a}^{*}$ in $\Omega$. For instance, if $\Omega$ is of rank $r$ it would be natural to have $\left(\Omega_{a_{1}}, \Omega_{a_{2}}, \ldots, \Omega_{a_{n}}\right)_{\Omega}=0$ for $n>r+1$ in which case $\Omega_{a_{1} a_{2} a_{3} \cdots a_{n}}=0$ for $n>r$ (see below).

If we compare the above formulas with those of the previous subsection we notice first that the master equations (4.17) and (4.50) are exactly dual. Then we notice that (4.44), (4.46), (4.47), and (4.49) are dual to (4.10), (4.14), (4.15), and (4.20) with the simple prescription that the Grassmann parities in the sign factors are changed one step, $\varepsilon \rightarrow \varepsilon+1$. The same is true for (4.53) and (4.21) except for an additional sign in the last 
line due to the fact that we have made use of a Jacobi identity which does not satisfy the simple rule above.

As an illustration of our formulas we consider now functions $f_{a}$ forming a quasigroup rank one theory in the antibracket sense. In this case we have (cf. [17)

$$
\Omega=\mathcal{C}^{a} f_{a}+\frac{1}{2} \mathcal{C}^{b} \mathcal{C}^{a} U_{a b}^{c} \mathcal{C}_{c}^{*}(-1)^{\varepsilon_{b}}
$$

where $(\Omega, \Omega)=0$ requires $\left(f_{a}, f_{b}\right)=U_{a b}^{c} f_{c}$ and

$$
U_{a b}^{d} U_{d c}^{e}(-1)^{\varepsilon_{b}}-\left(f_{a}, U_{b c}^{e}\right)(-1)^{\varepsilon_{b}}+\operatorname{cycle}(a, b, c)=0
$$

plus conditions on $\left(U_{a b}^{c}, U_{d e}^{f}\right)$. The latter conditions are satisfied if we impose the conditions

$$
\left(U_{a b}^{c}, U_{d e}^{f}\right)=0, \quad\left(\left(f_{d}, U_{a b}^{c}\right), U_{e f}^{g}\right)=0,
$$

which are stronger than what is required by $(\Omega, \Omega)=0$. In this case $\Omega_{a}$ may be chosen to be

$$
\Omega_{a}(\phi)=\lambda_{a}^{b}(\phi) \mathcal{C}_{b}^{*}(-1)^{\varepsilon_{b}}, \quad \lambda_{a}^{b}(0)=\delta_{a}^{b}
$$

where

$$
\left(\lambda_{a}^{b}(\phi), \lambda_{c}^{d}(\phi)\right)=0, \quad \Leftrightarrow \quad\left(\Omega_{a}(\phi), \Omega_{b}(\phi)\right)=0
$$

We require then

$$
\left(\Omega_{a}, \Omega_{b}, \Omega_{c}\right)_{\Omega}=0 \quad \Leftrightarrow \quad\left(\left\{\Omega_{a}, \Omega_{b}\right\}_{\Omega}, \Omega_{c}\right)=0,
$$

which allows for the choice $\Omega_{a b}=0$ since (4.59) makes the Poisson bracket $\left\{\Omega_{a}, \Omega_{b}\right\}_{\Omega}$ satisfy the Jacobi identities due to (3.8), which in turn makes (4.44) integrable without the need of a nontrivial $\Omega_{a b}$. All higher integrability conditions are then identically zero. Condition (4.59) is satisfied if we impose the conditions

$$
\left(\lambda_{a}^{b}(\phi), U_{d e}^{c}\right)=0, \quad\left(\lambda_{a}^{b},\left(\lambda_{c}^{d}, f_{e}\right)\right)=0 .
$$

All the above conditions specify quasigroups in an antibracket sense. We turn now to (4.44). We find then

$$
\begin{aligned}
& \left\{\Omega_{a}, \Omega_{b}\right\}_{\Omega}=\left(\Omega_{a},\left(\Omega, \Omega_{b}\right)\right)=-\lambda_{a}^{f} \lambda_{b}^{e} U_{e f}^{d} \mathcal{C}_{d}^{*}(-1)^{\varepsilon_{b}+\varepsilon_{e}+\varepsilon_{b} \varepsilon_{f}}- \\
& -\left(\lambda_{a}^{c}\left(f_{c}, \lambda_{b}^{d}\right)-\lambda_{b}^{c}\left(f_{c}, \lambda_{a}^{d}\right)(-1)^{\left(\varepsilon_{a}+1\right)\left(\varepsilon_{b}+1\right)}\right) \mathcal{C}_{d}^{*}(-1)^{\varepsilon_{c}+\varepsilon_{d}} .
\end{aligned}
$$

Eq.(4.44) may then be written as

$$
\partial_{a} \tilde{\lambda}_{b}^{c}+\partial_{b} \tilde{\lambda}_{a}^{c}(-1)^{\varepsilon_{a} \varepsilon_{b}}=(-1)^{\varepsilon_{a}} \tilde{\lambda}_{a}^{e} \tilde{\lambda}_{b}^{d}(-1)^{\left(\varepsilon_{b}+1\right)\left(\varepsilon_{e}+1\right)} \tilde{U}_{d e}^{c}(-1)^{\varepsilon_{c}+\varepsilon_{d}+\varepsilon_{e}} .
$$

where $\tilde{\lambda}_{a}^{b} \equiv W \lambda_{a}^{b}$ and $\tilde{U}_{a b}^{c} \equiv W U_{a b}^{c}$, where the differential operator $W$ satisfies the equation

$$
\partial_{a} W=W A d\left(G_{a}\right), \quad G_{a} \equiv \lambda_{a}^{b} f_{b}(-1)^{\varepsilon_{a}+\varepsilon_{b}}
$$

where in turn $A d(A) X \equiv(A, X)$ for any function $X$. The integrability condition of (4.63) is

$$
\partial_{a} G_{b}-\partial_{b} G_{a}(-1)^{\left(\varepsilon_{a}+1\right)\left(\varepsilon_{b}+1\right)}+\left(G_{a}, G_{b}\right)=0
$$


which is equivalent to (4.62).

One may note that

$$
\begin{aligned}
& Y_{a}(\phi)=\left(\Omega, \Omega_{a}\right)=\lambda_{a}^{b} f_{b}(-1)^{\varepsilon_{a}+\varepsilon_{b}}+ \\
& +\lambda_{a}^{b} \mathcal{C}^{d} U_{d b}^{c} \mathcal{C}_{c}^{*}(-1)^{\varepsilon_{a}}+\mathcal{C}^{b}\left(f_{b}, \lambda_{a}^{c}\right) \mathcal{C}_{c}^{*}(-1)^{\varepsilon_{c}} .
\end{aligned}
$$

If we assume $R(\phi)$ to have zero antibracket with the last terms, then (4.36) reduces to (3.28). It is amusing that in the quasigroup case the expressions (4.54), (4.57), and (4.65) for $\Omega, \Omega_{a}$, and $Y_{a}$ may be obtained from (4.22), (4.25), and (4.33) by the replacement $\mathcal{P}_{a} \rightarrow \mathcal{C}_{a}^{*}(-1)^{\varepsilon_{a}}$.

\section{Conclusions.}

We have considered antibrackets on symplectic manifolds and Poisson brackets on antisymplectic manifolds, as well as generating functions for these brackets. To every relation in the first case we have found a dual one in the second case and vice versa. In our presentation we have started with the simplest relations which we have successively generalized to finally end up with the remarkable master equations (4.17) and (4.49) representing generalized Maurer-Cartan equations. It might be useful to briefly summarize our results. If we consider a $(2 n, 2 n)$-dimensional manifold $\mathcal{E}$ it may be either a symplectic or an antisymplecic manifold. In the case $\mathcal{E}$ is a symplectic manifold we define a pre-antibracket or 2-antibracket by (2.1). This bracket satisfies all properties of a conventional antibracket except for the Jacobi identities (B.4) and Leibniz' rule (B.5). We claim that if the odd function $Q$ satisfies $\{Q, Q\}=0$ then we have a consistent set of generalized antibrackets starting from the 2-antibracket (2.1). The higher order antibrackets may be expressed in terms of the lower ones recursively. The general 3-antibracket is e.g. given by (3.15). Depending on the properties of $Q$ there is a certain level at which the higher order brackets terminate. All these brackets are interrelated in such a way that the Jacobi identities for (2.1) are not necessary for consistency. It is remarkable that just these brackets enter in a natural way in the master equation (4.17). In a way this demonstrates their importance. We have also given generating functions for these brackets: in section 3 for functions satisfying Lie algebra relations, and in section 4 for functions in arbitrary involutions in the original Poisson bracket. The 2-antibracket (2.1) satisfies the Jacobi identities only if the 3-antibracket (3.15) vanishes according to (3.14). This condition either restricts $Q$ or the class of functions to be considered. When the 3 -antibracket vanishes then all higher antibrackets vanish. Leibniz' rule (B.5) for the 2-antibracket leads then to further restrictions. In section 2 we showed that if we restrict to a class of functions satisfying an abelian algebra in the Poisson bracket then (2.1) is a true antibracket which can be non-degenerate on this submanifold of $\mathcal{E}$. Finally we have shown that all the above results are valid if $\mathcal{E}$ instead is an antisymplectic manifold on which we define the pre-Poisson bracket (2.2), i.e. all the above properties have their dual partners. In this latter case we have obtained a new type of generalized Poisson brackets involving higher Poisson brackets which are different from the Nambu brackets [14. The higher brackets are directly connected to the violations of the Jacobi identities. We have shown that these generalized Poisson brackets appear naturally in a new type of master equations for generalized Maurer-Cartan equations for open groups in an antibracket sense. 
Finally, we would like to mention that we believe there are also dual equations to the classical counterparts of the new $\operatorname{Sp}(2)$-antibrackets and $\operatorname{Sp}(2)$ master equations introduced in [18].

\section{Acknowledgments}

I.A.B. would like to thank Lars Brink for his very warm hospitality at the Institute of Theoretical Physics, Chalmers and Göteborg University. The work is partially supported by INTAS-RFBR grant 95-0829. The work of I.A.B. is also supported by INTAS grant 96-0308 and by RFBR grants 96-01-00482, 96-02-17314. I.A.B. and R.M. are thankful to the Royal Swedish Academy of Sciences for financial support.

\section{Appendix A}

\section{Defining properties of the conventional Poisson bracket.}

The defining properties of the Poisson bracket, $\{f, g\}$, for functions $f, g$ on a manifold $\mathcal{S}$ are

1) Grassmann parity

$$
\varepsilon(\{f, g\})=\varepsilon_{f}+\varepsilon_{g}
$$

2) Symmetry

$$
\{f, g\}=-\{g, f\}(-1)^{\varepsilon_{f} \varepsilon_{g}}
$$

3) Linearity

$$
\{f+g, h\}=\{f, h\}+\{g, h\}, \quad\left(\varepsilon_{f}=\varepsilon_{g}\right) .
$$

4) Jacobi identities

$$
\{f,\{g, h\}\}(-1)^{\varepsilon_{f} \varepsilon_{h}}+\operatorname{cycle}(f, g, h) \equiv 0 .
$$

5) Leibniz' rule

$$
\{f g, h\}=f\{g, h\}+\{f, h\} g(-1)^{\varepsilon_{g} \varepsilon_{h}} .
$$

6) For any odd/even parameter $\lambda$ we have

$$
\{f, \lambda\}=0 \quad \text { any } f \in \mathcal{S} \text {. }
$$

The Poisson bracket can only be nondegenerate if the dimension of the even subspace of

$\mathcal{S}$ is even. ( $\mathcal{S}$ is then a symplectic manifold.) If $z^{A}$ are independent coordinates on $\mathcal{S}$ then we have explicitly $\left(\partial_{A} \equiv \partial / \partial z^{A}\right)$

$$
\{f(z), g(z)\}=f(z) \overleftarrow{\partial}_{A} \omega^{A B}(z) \partial_{B} g(z)
$$


where $\omega^{A B}(z)$ satisfies $\left(\varepsilon_{A} \equiv \varepsilon\left(z^{A}\right)\right)$

$$
\begin{aligned}
& \omega^{A B}(z)=-(-1)^{\varepsilon_{A} \varepsilon_{B}} \omega^{B A}(z), \quad \varepsilon\left(\omega^{A B}(z)\right)=\varepsilon_{A}+\varepsilon_{B} \\
& \omega^{A D}(z) \partial_{D} \omega^{B C}(z)(-1)^{\varepsilon_{A} \varepsilon_{C}}+\operatorname{cycle}(A, B, C)=0 .
\end{aligned}
$$

The last equalities follow from the Jacobi identities (A.4).

\section{Appendix B}

\section{Defining properties of the conventional antibracket.}

The defining properties of the antibracket $(f, g)$ for functions $f, g$ on a manifold $\mathcal{A}$ are

1) Grassmann parity

$$
\varepsilon((f, g))=\varepsilon_{f}+\varepsilon_{g}+1
$$

2) Symmetry

$$
(f, g)=-(g, f)(-1)^{\left(\varepsilon_{f}+1\right)\left(\varepsilon_{g}+1\right)}
$$

3) Linearity

$$
(f+g, h)=(f, h)+(g, h), \quad\left(\varepsilon_{f}=\varepsilon_{g}\right) .
$$

4) Jacobi identities

$$
(f,(g, h))(-1)^{\left(\varepsilon_{f}+1\right)\left(\varepsilon_{h}+1\right)}+\operatorname{cycle}(f, g, h) \equiv 0 .
$$

5) Leibniz' rule

$$
(f g, h)=f(g, h)+(f, h) g(-1)^{\varepsilon_{g}\left(\varepsilon_{h}+1\right)} .
$$

6) For any odd/even parameter $\lambda$ we have

$$
(f, \lambda)=0 \quad \text { any } f \in \mathcal{A} .
$$

The antibracket can only be nondegenerate if $\mathcal{A}$ is a supermanifold with the dimension $(n, n)$. ( $\mathcal{A}$ is then an antisymplectic manifold.) If $z^{A}$ are independent coordinates on $\mathcal{A}$ then we have explicitly

$$
\{f(z), g(z)\}=f(z) \overleftarrow{\partial}_{A} E^{A B}(z) \partial_{B} g(z)
$$

where $E^{A B}(z)$ satisfies

$$
\begin{aligned}
& E^{A B}(z)=-(-1)^{\left(\varepsilon_{A}+1\right)\left(\varepsilon_{B}+1\right)} E^{B A}(z), \quad \varepsilon\left(E^{A B}(z)\right)=\varepsilon_{A}+\varepsilon_{B}+1, \\
& E^{A D}(z) \partial_{D} E^{B C}(z)(-1)^{\left(\varepsilon_{A}+1\right)\left(\varepsilon_{C}+1\right)}+\operatorname{cycle}(A, B, C)=0 .
\end{aligned}
$$

The last equalities follow from the Jacobi identities (B.4). 


\section{References}

[1] J. A. Schouten, Koninklijke Nederlandse Akademie van Wetenschappen, Series A, Proceedings 2, 449 (1940); On the differential operators of first order in tensor calculus, in Convegno Internazionale di Geometria Differenziale, Italy, Sept. 20-26, 1923, Edizioni Cremonese delle Casa Editrice Perrella, Rome, (1954);

C. Buttin, C.r. Acad. Sci. Paris 269, A87 (1969);

J. Zinn-Justin, Renormalization of gauge theories, in Trends in Elementary Particle Theory, Lecture Notes in Physics (eds. Rollnik and Dietz), vol 37, SpringerVerlag, Berlin, 1975;

E. Witten, Modern Phys. Lett. A5, 487 (1990).

[2] I. A. Batalin and G. A. Vilkovisky, Phys. Lett. B102, 27 (1981); Phys. Rev. D28, 2567 (1983).

[3] I. A. Batalin and R. Marnelius, Phys. Lett. B434, 312 (1998).

[4] O. M. Khudaverdian and A. P. Nersessian, J. Math. Phys. 37, 3713 (1996); A. P. Nersessian, Preprint JINR E2-93-225 (hep-th/9306111); Preprint JINR E2-93-358.

[5] A. P. Nersessian and P. H. Damgaard, Phys. Lett. B355, 150 (1995).

[6] M. A. Grigoriev, A. M. Semikhatov, and I. Yu. Tipunin, Gauge Symmetries of the Master Action, hep-th/9804156.

[7] I. A. Batalin and R. Marnelius, Open groups of constraints - Integrating arbitrary involutions, hep-th/9806114, (To be published in Phys. Lett. B.)

[8] D. V. Volkov, A. I. Pashnev, V. A. Soroka, and V. I. Tkach, JETP Lett. 44, 70 (1986); Teor. Mat. Fiz. 79, 117 (1989);

V. A. Soroka, Lett. Math. Phys. 17, 201 (1989).

[9] O. M. Khudaverdian, J. Math. Phys. 32, 1934 (1991);

O. M. Khudaverdian and A. P. Nersessian, J. Math. Phys. 32, 1938 (1991).

[10] I.A. Batalin and I.V. Tyutin, Nucl. Phys. B381, 619 (1992); Phys. Lett. B302, 47 (1993); ibid B317, 354 (1993); Int. J. Mod. Phys. A8, 3757 (1993).

[11] M. Alexandrov, A. Schwarz and O. Zaboronsky, and M. Kontsevich, Int. J. Mod. Phys. A12, 1405 (1997).

[12] I. A. Batalin, R. Marnelius and A. M. Semikhatov, Nucl. Phys. B446, 249 (1995); I. A. Batalin and R. Marnelius, Nucl. Phys. B465, 521 (1996).

[13] J.-L. Koszul, Astérisque, hors serie 257 (1985);

F. Akman, q-alg/9506027;

J. Alfaro and P. H. Damgaard, Phys. Lett. B369, 289 (1996);

K. Bering, P. H. Damgaard, and J. Alfaro Nucl. Phys. B478, 459 (1996);

I. A. Batalin, K. Bering, and P. H. Damgaard, Phys. Lett. B389, 673 (1996).

[14] Y. Nambu, Phys. Rev. D7, 2405 (1973). 
[15] I. A. Batalin and G. A Vilkovisky, Phys. Lett. B69, 309 (1977);

E. S. Fradkin T. E. Fradkina, Phys. Lett. B72, 343 (1978);

I. A. Batalin and E. S. Fradkin, Phys. Lett. B122, 157 (1983).

[16] I. A. Batalin, J. Math. Phys. 22, 1837 (1981).

[17] I. A. Batalin and R. Marnelius, Nucl. Phys. B511, 495 (1998).

[18] I. A. Batalin and R. Marnelius, Quantum Sp(2)-antibrackets and open groups, hep-th/9809208. 